machen. Namentlich die politischen Eliten in diesen Ländern sowie die Imperialismustheoretiker, nicht aber die Dependenztheoretiker, neigen immer wieder zu diesem einseitigen bzw. sie legitimierenden Fehlansatz.

Trotz dieser Kritik im einzelnen, die bei dem ersten Versuch, ein solches Unternehmen zu starten, nahezu unvermeidlich herausgefordert wird, will der Rezensent nicht verhehlen, daß er über weite Strecken mit großem Interesse und Genuß in den drei Büchern gelesen hat. Als erste, etwas ausführlichere Information über die einzelnen Länder der Dritten Welt gehört dieses Handbuch in jede Bibliothek. Es ist bedauerlich, daß der doch recht stattliche Preis es vielen Studenten wohl nicht ermöglichen wird, sich diese Bücher anzuschaffen.

Rolf Hanisch

Hans-Jürgen Puhle, Hrsg.:

\title{
Lateinamerika. Historische Realität und Dependencia-Theorien
}

Hoffmann \& Campe, Hamburg 1977, 240 S., 32,- DM

$\mathrm{Daß}$ die Dependencia-Theorien ein starkes Empiriedefizit aufweisen, ist schon lange bekannt und wird auch von so manchen Dependenz-Autoren beklagt (und von anderen zur besonderen Tugend einer angeblich anti-bürgerlichen Wissenschaft hochstilisiert). Daß dem so offenkundig empfundenen Mangel bisher sehr wenig abgeholfen wurde, mag wohl daran gelegen haben, daß bei einem wissenschaftlichen Neuansatz, dem man modische Qualitäten nicht absprechen kann, sich mit großen theoretischen Würfen mehr Meriten verdienen lassen als mit mühevoller empirischer Kleinarbeit, selbst wenn mit diesen globalen Entwürfen nicht selten alter Wein in neuen Schläuchen verkauft wird (sehr deutlich hierzu de Imaz). Um so erfreulicher sollte es sein, wenn sich Historiker mit der ihnen eigenen Akribie daran machen, den Dependencia-Theorien ihr bislang noch fehlendes empirisches Unterfutter zu liefern, bzw. den Theoretikern die Chance bieten, sich zu korrigieren.

Das Verfahren ist jedoch schwieriger, als es zunächst den Anschein hat. Die Dependencia-Theorie gibt es nicht. Statt dessen hat sich der empirisch ausgerichtete Wissenschaftler mit einer Vielzahl von Ansätzen auseinanderzusetzen, die sich alle irgendwie am Begriff der Abhängigkeit orientieren, diesen aber jeweils höchst unterschiedlich fassen, und die darüber hinaus häufig mit einem unterdeterminierten und zuweilen auch schon formal untauglichen, da tautologischen Dependenzbegriff arbeiten. Angesichts der theoretischen Vielfalt stellt sich bei einem Versuch einer empirischen Überprüfung die Frage, welchen Dependenzansatz man überprüfen will. In anderen Worten, man müßte die Autoren eines Sammelbandes entweder auf die Überprüfung eines bestimmten Ansatzes verpflichten oder in einem Seminar (das in diesem Falle ja abgehalten wurde) ein generelles Dependenzverständnis herausarbeiten, das gewissermaßen den gemeinsamen Fundus aller oder der Mehrzahl der Dependenzansätze darstellt, und das dann einer empirischen Überprüfung unterzogen werden kann.

Genau dies ist jedoch nicht geschehen, mit dem Resultat, daß die Kritik und die Korrekturen an der Dependenztheorie, die es gar nicht, genauso diffus ausgefallen sind wie die Vielzahl der theoretischen Ansätze. Mit diesem Verfahren läßt sich gewiß nicht feststellen, ob die Kategorie der Abhängigkeit für die historische Forschung brauchbar ist, was nach Meinung des Herausgebers eigentlich Aufgabe dieses Buches gewesen wäre. 
Hinzu kommen noch weitere Schwierigkeiten. Es scheint, daß vor allem Autoren des zweiten Teils des Bandes, in dem bestimmte historische Themen bearbeitet werden, angesichts der Vielfalt der Dependenzbegriffe aufgegeben haben und daher auch gar nicht mehr deutlich machen, was sie eigentlich überprüfen wollen. Klaus Lindenberg („Militär und Abhängigkeit in Latainamerika. Fremdbestimmende Faktoren seiner institutionellen Entwicklung, seines professionellen Rollenverständnisses und seines politischen Verhaltens") legt zwar einen der informativsten Beiträge zur Rolle des Militärs in Lateinamerika vor, der bisher in deutscher Sprache erschienen ist, stellt aber den Bezug zur Theorie der Abhängigkeit allein mit dem Begriff der Fremdbestimmung her, was auf den theoretischen Anspruch der Dependencia-Ansätze weder eingeht noch eingehen soll und diese damit beinahe auf den Status eines banalen "truism" reduziert. Konrad Stentzel („Die Entstehung des republikanischen Venezuela, oder: die Grenzen des kapitalistischen Fortschritts. Bemerkungen zum Scheitern von ,Modernisierung' in den Agrargesellschaften Lateinamerikas") bietet eine interessante Interpretation für das Scheitern der kapitalistischen Entwicklungsstrategie der venezolanischen Eliten im frühen 19. Jahrhundert, beschränkt aber den Bezug zur Dependencia auf eine abschließende Seite, in der einige zutreffende Bemerkungen zum generellen Charakter der DependenciaAnsätze gemacht werden, was wiederum offen läßt, was denn hier überprüft worden ist.

Die Beiträge von Reinhard Liehr („Entstehung, Entwicklung und sozialökonomische Struktur der hispanoamerikanischen Hacienda“) und Horst Pietschmann („Dependencia-Theorie und Kolonialgeschichte. Das Beispiel des Warenhandels der Distriktsbeamten im kolonialen Hispanoamerika") geben hingegen deutlich an, was und wen sie überprüfen wollen. Ihre Forschungsergebnisse stellen Korrekturen an dem Bild der politischen OOkonomie der Kolonialepoche dar, das einige Dependencia-Autoren gezeichnet haben (Armando Córdova, André Gunder Frank), lassen aber kaum den Schluß zu, daß "aus der Sicht des Historikers die Dependencia-Theorie(en) weder in allgemeiner Hinsicht noch in bezug auf Einzelaspekte des geschichtlichen Lebens geeignet ist, die kolonialzeitliche historische Entwicklung Lateinamerikas zu erklären" (Pietschmann, S. 164 f.). Abgesehen davon, daß Pietschmann Konsequenz mit Funktionalität verwechselt, wenn er die Dysfunktionalität des von Beamten kontrollierten Warenhandels für die spanischen Kolonialinteressen als Beleg gegen die Dependenztheorie(en) anführt, und unterstellt, daß Dependenz eine totale, alle Aspekte der kolonialen OKkonomie umfassende Fremdbestimmung im Interesse der Kolonialmacht bedeute, liegt hier noch ein weiteres Mißverständnis vor, das allerdings von André Gunder Frank, auf den Pietschmann sich offenbar stark bezieht, in die Welt gesetzt worden ist: wenn er nachweist, daß nur wenige Teilbereiche der kolonialen Wirtschaft nach kapitalistischen Prinzipien betrieben wurden, dann kann er damit zwar Frank kritisieren (was übrigens auch schon andere Dependenzautoren mit derselben Begründung getan haben), er bietet aber gleichzeitig mit dieser Argumentation eine Bilderbuchillustration für etwas, was von anderen Dependenzautoren mit dem Begriff der „strukturellen Heterogenität" beschrieben worden ist. Und Reinhard Liehr hat natürlich völlig recht, wenn er in Kritik an einigen Dependenzautoren feststellt, daß „im Hinblick auf die mexikanische und peruanische Hochlandhacienda des 17. bis 19. Jahrhunderts die Generalhypothese der Dependencia abstrakt und nicht operationalisierbar (bleibt)“ (S. 134), doch trifft er damit nur die Auffassung von Dependenz, die Abhängigkeit 
als totale Produktionsausrichtung auf die Bedürfnisse der Kolonialmacht versteht. Seine Kritik fügt sich jedoch sehr gut in eine Dependenzauffassung ein, die gerade die strukturelle Heterogenität als kennzeichnendes Merkmal abhängiger Gesellschaften sieht.

Die beiden letztgenannten historischen Detailuntersuchungen, aber auch der einleitende Artikel von Hans-Jürgen Puhle („Dependencia - eine Kategorie der historischen Lateinamerikaforschung?") und der wirtschaftswissenschaftlich orientierte Beitrag von Hermann Sautter („Unterentwicklung und Abhängigkeit als Ergebnisse wirtschaftlicher Verflechtung. Zum ökonomischen Aussagewert der Dependencia-Theorie") machen es sich aber auch in anderer Hinsicht mit ihrer Einschätzung der Dependencia-Ansätze zu leicht. Wenn die Autoren die ihnen vertrauten Kategorien (bei den Historikern: Kolonialismus, koloniale Situation [Balandier], duale Gesellschaft) mit den offensichtlich weniger vertrauten Begriffen der Dependencia-Ansätze kontrastieren, kommen sie mit schöner Regelmäßigkeit zu dem Schluß, daß diejenigen Kategorien der Dependencia-Forschung brauchbar seien, die sich mit den etablierten Begriffen der historischen Forschung decken, daß der Rest aber wirr und/oder banal sei. Dieses Verfahren signalisiert ein deutliches Perzeptionsproblem einer Wissenschaft, für die offenbar "alles schon einmal dagewesen ist". Daß es sich um ein Perzeptionsproblem einer etablierten Wissenschaft handelt, bestätigt sich, wenn man Behauptungen findet wie die, daß „strukturelle Heterogenität" nichts anderes bedeute als die „empirische Gleichzeitigkeit des Ungleichzeitigen" (Puhle, S. 18): genau das ist damit nicht gemeint, aber man vermag offenbar nur Vertrautes zu entdecken. Wenn Sautter mit gutem empirischem Material einige von ihm aus der Literatur herausdestillierte Grundannahmen der Dependencia-Schule kritisiert und an einer Stelle ausgerechnet Cardoso und Faletto als Kronzeugen für seine Kritik zitiert (S. 42), ist das ein recht merkwürdiges Verfahren, sich mit der Dependencia-Schule auseinanderzusetzen. Eine empirische Überprüfung einer theoretischen Richtung sollte nicht so offensichtlich deren komplexere Ansätze ausklämmern, denen man mit leichtem Geschütz nicht so ohne weiteres beikommen kann.

Der einzige Beitrag, der sich auf faire Weise kritisch mit den Dependencia-Ansätzen auseinandersetzt, ist der von Philip J. O’Brien („Zur Kritik lateinamerikanischer Dependencia-Theorien“), in dem, ähnlich wie bei Evers und v. Wogau, die begrifflichen Unschärfen und empirischen Schwachstellen vieler DependenciaAnsätze herausgearbeitet werden. Es ist gewiß zuzugeben, daß viele Dependenzautoren den Kritikern, die sie widerlegen wollen, selbst die besten Argumente liefern, und ein der Genauigkeit verpflichteter Historiker hat mit einem Autor wie A. G. Frank sicherlich keine Mühe. Eine Sichtung dessen, was an den DependenciaAnsätzen brauchbar ist, erfordert Geduld; wer diese Geduld aufbringt, kann durchaus zu neuen theoretischen Erkenntnissen und Einsichten kommen.

Trotz aller Kritik handelt es sich aber bei diesem Sammelband um ein nützliches und notwendiges Buch. Die Schwächen vieler Dependencia-Ansätze, ihre häufigen begrifflichen Unschärfen und ihre empirische Schludrigkeit, werden sehr klar herausgearbeitet, und die der Dependencia-Schule verpflichteten Autoren täten gut daran, dies als Herausforderung zu empfinden. Außerdem stehen alle Detailuntersuchungen auf einem hohen Niveau und bieten, sieht man einmal von der mißglückten Auseinandersetzung mit der Dependencia-Theorie ab, vorzügliche Analysen zu einigen Aspekten der lateinamerikanischen Geschichte. Andreas Boeckh 